\title{
A new achievement scalarizing function based on parameterization in multiobjective optimization
}

\author{
Yury Nikulin • Kaisa Miettinen • \\ Marko M. Mäkelä
}

Published online: 11 August 2010

(C) Springer-Verlag 2010

\begin{abstract}
This paper addresses a general multiobjective optimization problem. One of the most widely used methods of dealing with multiple conflicting objectives consists of constructing and optimizing a so-called achievement scalarizing function (ASF) which has an ability to produce any Pareto optimal or weakly/properly Pareto optimal solution. The ASF minimizes the distance from the reference point to the feasible region, if the reference point is unattainable, or maximizes the distance otherwise. The distance is defined by means of some specific kind of a metric introduced in the objective space. The reference point is usually specified by a decision maker and contains her/his aspirations about desirable objective values. The classical approach to constructing an ASF is based on using the Chebyshev metric $L_{\infty}$. Another possibility is to use an additive ASF based on a modified linear metric $L_{1}$. In this paper, we propose a parameterized version of an ASF. We introduce an integer parameter in order to control the degree of metric flexibility varying from $L_{1}$ to $L_{\infty}$. We prove that the parameterized ASF supports all the Pareto optimal solutions. Moreover, we specify conditions under which the Pareto optimality of each solution is guaranteed. An illustrative example for the case of three objectives and comparative analysis of parameterized ASFs with different values of the parameter are given. We show that the parameterized ASF provides the decision maker with flexible and advanced tools
\end{abstract}

\footnotetext{
Y. Nikulin $(\varangle) \cdot$ M. M. Mäkelä

Department of Mathematics, University of Turku, 20014 Turku, Finland

e-mail: yurnik@utu.fi

M. M. Mäkelä

e-mail: makela@utu.fi

K. Miettinen

Department of Mathematical Information Technology, University of Jyväskylä,

40014 Jyväskylä, Finland

e-mail: kaisa.miettinen@jyu.fi
} 
to detect Pareto optimal points, especially those whose detection with other ASFs is not straightforward since it may require changing essentially the reference point or weighting coefficients as well as some other extra computational efforts.

Keywords Multiobjective optimization - Achievement function · Parameterization · Pareto optimal solutions · Multiple criteria decision making

\section{Introduction}

Many real-life optimization problems can hardly be considered as properly formulated without taking into account their multiple objective nature. This fact commonly accepted by many experts explains a permanently growing interest in the area of multiobjective optimization. Considering multiple conflicting objectives is advantageous in comparison with optimizing one single objective only. It is well known that a solution which is optimal with respect to one single objective may be arbitrarily bad with respect to other objectives and thus will be unacceptable for the decision maker (DM). While optimizing one objective usually leads to one optimal solution, multiobjective optimization involves dealing with a set of Pareto optimal solutions (alternatives) that provide different trade-offs between several conflicting objectives. Usually these objectives represent various interests. For example, for some transportation problems one goal may be oriented on passenger comfort while another one may represent convenience for the transport company. Thus, finding a compromise between several goals may positively influence interests of all participants involved. The primary goal of multiobjective optimization is to optimize simultaneously several conflicting objectives in order to find Pareto optimal solutions acceptable for the DM. Optimality is usually understood in the sense of Pareto optimality, but other optimality principles (lexicographic, Smale, Slater, Geoffrion, Borwein, Condorcet etc., see, e.g. Sawaragi et al. 1985) can also be used.

In multiobjective optimization, vectors are regarded as optimal if their components cannot be improved without deteriorating the others. This concept was first introduced by Pareto (1909). The Pareto optimality principle generally describes an equilibrium situation such that the value of no objective for any Pareto optimal solution can be improved without getting the value of another objective deteriorated.

There is a large variety of methods suggested for solving multiobjective optimization problems (see, e.g., Ehrgott 2000; Miettinen 1999; Sawaragi et al. 1985; Steuer 1986). Many of these methods are based on a scalarization approach. Via scalarization, the problem is transformed into a single objective optimization problem involving possibly some parameters or additional constraints. In most scalarizing functions, additional information is requested from the DM about her/his individual preferences and further taken into consideration. After the scalarization phase, the widely developed theory and methods of single objective optimization are available.

Multiobjective optimization methods utilize different scalarizing functions in different ways. The input requested from the DM may consist of trade-off information, marginal rates of substitution or desirable objective function values (see ++e.g. Luque et al.). Furthermore, the scalarization may be performed once or repeatedly as a part 
of an iterative process. When methods are introduced in the literature, the optimality of the results produced is usually proved. On the other hand, it is not so common to justify why some specific form of scalarization is used. The choice of the particular scalarization approach has to be done very carefully, since different scalarizations typically produce different Pareto optimal solutions.

One of the most widely used approaches of dealing with multiple conflicting objectives involves constructing and optimizing a so-called achievement scalarizing function (ASF) (Miettinen 1999; Wierzbicki 1980, 1986b) which has an ability to produce any (properly) Pareto optimal or weakly Pareto optimal solution. The ASF minimizes the distance from the reference point (specified by the DM) to the feasible region, if the reference point is unattainable, or maximizes the distance otherwise. The distance is defined by means of some appropriate metric introduced in the objective space. The classical approach to constructing an ASF is based on using Chebyshev $L_{\infty}$ and linear $L_{1}$ metrics (see, e.g. Miettinen 1999).

Our work is inspired by Ruiz et al. (2008) where an achievement scalarizing function based on using a modified linear metric was proposed. As it was truly noticed Ruiz et al. (2008), there might be some situations where the DM may want to minimize not the maximal unwanted deviation from the reference point, but the weighted sum of unwanted deviations instead. We enhance this idea of Ruiz et al. (2008) by assuming that the DM's wishes can be even more complicated involving more advanced scalarization mechanisms. So, in our paper we extend some general ideas of Ruiz et al. (2008) and propose a parameterized version of an ASF. We introduce an integer parameter in order to control the degree of metric flexibility varying from $L_{1}$ to $L_{\infty}$. We prove that the parameterized ASF is able to detect any Pareto optimal solutions. Moreover, we specify conditions under which the Pareto optimality of each solution produced by the parameterized ASF is guaranteed.

The paper is organized as follows: Sect. 2 is devoted to the main definitions and concepts. In Sect. 3 we briefly give a description for a class of methods based on the concept of a reference point. In Sect. 4 we propose a new approach to creating an achievement scalarizing function which is based on parameterization. Theoretical justification of the approach is given. An illustrative example for the case of a threedimensional objective space is presented in Sect. 5. Final remarks and open questions are presented in Sect. 6.

\section{Preliminaries and basic definitions}

Let $X$ be an arbitrary set of feasible solutions or a set of decision vectors. Let a vector valued function $f: X \rightarrow \mathbf{R}^{m}$ consisting of $m \geq 2$ objective functions be defined on the set of feasible solutions:

$$
f(x)=\left(f_{1}(x), f_{2}(x), \ldots, f_{m}(x)\right) .
$$

Without loss of generality we assume that every objective function is subject to be minimized on the set of feasible solutions:

$$
f_{i}(x) \longrightarrow \min _{x \in X}, \quad i \in N_{m}=\{1,2, \ldots, m\}
$$


Further, throughout the paper we will refer to $\mathbf{R}^{m}$ as an objective space and vector $f(x)$ as an objective vector.

We also assume that

(i) every objective function $f_{i}$ is a lower semicontinuous function;

(ii) $X$ is a nonempty compact set.

We denote by

$$
M^{i}(X)=\arg \min _{x \in X} f_{i}(x), \quad i \in N_{m}
$$

a set of minima of the $i$-th objective function.

Evidently, if

$$
\bigcap_{i=1}^{m} M^{i}(X) \neq \emptyset
$$

then there exists at least one solution which delivers a minimum for all objectives. Such a solution can be called an ideal solution. An optimization problem which does not contain ideal solutions is called non-degenerate and objectives are at least partly conflicting. Simultaneous optimization of several objectives for non-degenerate multiobjective optimization problems is not a straightforward task, and we need to define optimality for such problems. In what follows, we consider non-degenerate problems.

The following definition formalizes the concept of Pareto optimality:

Definition 1 A decision vector $x^{*} \in X$ is Pareto optimal if there does not exist another $x \in X$ such that $f_{i}(x) \leq f_{i}\left(x^{*}\right)$ for all $i \in N_{m}$ and $f_{j}(x)<f_{j}\left(x^{*}\right)$ for at least one index $j$.

We can denote the set of Pareto optimal decision vectors as $P^{m}(X)$. Furthermore, the set $\left\{f(x) \in \mathbf{R}^{m}: x \in P^{m}(X)\right\}$ is called the Pareto frontier. For two vectors $a, b \in \mathbf{R}^{m}$, we write $a \leq b$ if $a_{i} \leq b_{i}$ for all $i \in N_{m}$. Then we say that one vector $a$ dominates another vector $b$ if $a \leq b$ and $a \neq b$. Thus, the set of Pareto optimal solutions is simply a subset of feasible solutions whose images are non-dominated in the objective space.

The optimality in a multiobjective case can be introduced in different ways. The following definition was firstly proposed by Slater (1950):

Definition 2 A decision vector $x \in X$ is weakly Pareto optimal if there does not exist another $x \in X$ such that $f_{i}(x)<f_{i}(x)$ for all $i \in N_{m}$.

We can denote the set of weakly Pareto optimal decision vectors or Slater set as $S l^{m}(X)$. For two vectors $a, b \in \mathbf{R}^{m}$, we write $a<b$ if $a_{i}<b_{i}$ for all $i \in N_{m}$. Then we say that one vector $a$ strictly dominates another vector $b$ if $a_{i}<b_{i}$ for all $i \in N_{m}$. Thus, the set of weakly Pareto optimal solutions is a subset of feasible solutions whose images are strictly non-dominated in the objective space. An objective vector $f(x)$ is (weakly) Pareto optimal if the corresponding decision vector $x$ is (weakly) Pareto optimal.

Under the assumptions (i)-(ii) mentioned earlier in the problem formulation, we know that the set of Pareto optimal solutions is non-empty, i.e., there always exists at 
least one Pareto optimal solution (see Sawaragi et al. 1985, Corollary 3.2.1). Obviously, the set of Pareto optimal solutions is a subset of weakly Pareto optimal solutions.

Even though the concept of Pareto optimality corresponds to the intuitive idea of a compromise and rational behavior of the DM, we deal with both Definitions 1 and 2 because weakly Pareto optimal solutions are sometimes computationally more convenient to produce than Pareto optimal solutions.

Lower and upper bounds on objective values of all Pareto optimal solutions are given by the ideal and nadir objective vectors $f^{I}$ and $f^{N}$, respectively. The components $f_{i}$ of the ideal (nadir) objective vector $f^{I}=\left(f_{1}^{I}, \ldots, f_{m}^{I}\right)\left(f^{N}=\left(f_{1}^{N}, \ldots, f_{m}^{N}\right)\right)$ are obtained by minimizing (maximizing) each of the objective functions individually subject to the set of Pareto optimal solutions:

$$
\begin{aligned}
f_{i}^{I} & =\min _{x \in P^{m}(X)} f_{i}(x), \quad i \in N_{m}, \\
f_{i}^{N} & =\max _{x \in P^{m}(X)} f_{i}(x), \quad i \in N_{m} .
\end{aligned}
$$

For calculating the ideal objective vector, minimization over the set of Pareto optimal solutions can be replaced by minimization over the set of all feasible solutions. Such a replacement allows to calculate the ideal objective vector without having explicit information about the entire Pareto optimal set. Due to this, in fact, the concept of an ideal objective vector coincides with the concept of an ideal solution introduced earlier. Unfortunately, calculating the nadir objective vector is not that simple. The upper bounds of the Pareto optimal set, that is, the components of a nadir objective vector $f^{N}$ are hard to compute for problems with more than two objectives. The nadir objective vector can, however, be estimated from a payoff table, but this estimation might be not very reliable (see, e.g. Miettinen 1999). Recently, some approaches have been proposed as efficient approximation techniques to obtain a good approximation of the nadir objective vector (see, e.g. Deb and Miettinen 2010).

Sometimes, a vector strictly better than $f^{I}$ is required. This vector is called a utopian objective vector and denoted by $f^{U}$. In practice, the components of the utopian objective vector are calculated by subtracting some small positive scalar from the components of the ideal objective vector:

$$
f^{U}=f^{I}-\varepsilon \cdot 1_{(m)},
$$

where $1_{(m)}$ is an $m$-dimensional vector of all ones and $\varepsilon$ is a small positive parameter.

There exists a large variety of methods proposed to deal with problems involving multiple objectives. As mentioned in the introduction, a widely used approach for solving multiobjective optimization problems is scalarization, that is, converting the multiple objectives together with possible preference information into one scalarized objective. Two major requirements are set for a scalarization function in order to provide method completeness (Sawaragi et al. 1985):

- it should be able to cover the entire set of Pareto optimal solutions, and

- every solution found by means of scalarization should be (weakly) Pareto optimal. 
The mechanism of scalarization is a key issue behind many different methods for multiobjective optimization reported in the literature.

The simplest approach is a linear scalarization, also known as the weighting method (Miettinen 1999; Steuer 1986). This scalarization satisfies the two aforementioned requirements for convex optimization problems, but not for nonconvex and discrete unsupported cases. A major incremental complication of these cases is that not all Pareto optimal solutions are reachable as optimal solutions by means of linear scalarization. Such solutions are referred to as unsupported solutions in the literature (see, e.g. Steuer 1986).

A scalarization approach which is applicable for both convex and nonconvex problems is the minimization of some sort of distance from an ideal solution. As we already mentioned earlier, in the case of conflicting objectives, the ideal objective vector is not feasible, but it can serve as a reference point, with a goal to find solutions which are as close as possible to the ideal values with respect to the chosen distance measure. An example of this approach is the so-called compromise programming, also known as a method of global criterion, where the $L_{p}$ metric $(1 \leq p \leq \infty)$ can be used to measure the distance. If weighting coefficients are used in the metric, we get a so-called method of weighted metrics or weighted compromise programming. The two requirements set on scalarization functions are satisfied only if $L_{\infty}$, that is, the Chebyshev metric is used with the utopian objective vector as a reference point. For more advanced details on properties of compromise programming and $L_{p}$ problems, see Miettinen (1999) and references therein.

The usage of compromise programming has some limitations. One of the most important restrictions is that the usage of the ideal objective vector does not incorporate the preference information. To enable the DM's preferences to be included, one can use a reference point consisting of aspiration levels instead of the ideal objective vector. If we ask the DM to specify the reference point, we can take her/his preferences into account and hopefully generate more satisfactory solutions. The reference point reflects the DM's estimations about the desirable objective values. However, if we allow the DM to set the reference point, we must replace the $L_{p}$ metrics as distance measures by achievement scalarizing functions. In this way we can guarantee that the method works for both achievable and inachievable reference points Miettinen (1999).

In what follows, we concentrate on reference point-based scalarizing functions.

\section{Reference point-based approaches and achievement scalarizing functions}

In reference point based methods (see, e.g. Wierzbicki 1980, 1986b, 1999), the DM specifies a reference point $f^{R}$ consisting of desirable or reasonable aspiration levels $f_{i}^{R}$ for each objective function $f_{i}, i \in N_{m}$. This reference point only indicates what kind of objective function values the DM prefers.

Achievement scalarizing functions (ASFs) have been introduced by Wierzbicki (1980). The scalarized problem is given by

$$
\min _{x \in X} s_{R}(f(x)) .
$$


Certain properties of ASFs guarantee that problem (1) yields Pareto optimal solutions.

Definition 3 Wierzbicki (1986b) An ASF $s_{R}: \mathbf{R}^{m} \rightarrow \mathbf{R}$ is said to be

1. Increasing,

if for any $y^{1}, y^{2} \in \mathbf{R}^{m}, y_{i}^{1} \leq y_{i}^{2}$ for all $i \in N_{m}$, then $s_{R}\left(y^{1}\right) \leq s_{R}\left(y^{2}\right)$.

2. Strictly increasing,

if for any $y^{1}, y^{2} \in \mathbf{R}^{m}, y_{i}^{1}<y_{i}^{2}$ for all $i \in N_{m}$, then $s_{R}\left(y^{1}\right)<s_{R}\left(y^{2}\right)$.

3. Strongly increasing,

if for any $y^{1}, y^{2} \in \mathbf{R}^{m}, y_{i}^{1} \leq y_{i}^{2}$ for all $i \in N_{m}$ and $y^{1} \neq y^{2}$, then $s_{R}\left(y^{1}\right)<s_{R}\left(y^{2}\right)$.

Obviously, any strongly increasing ASF is also strictly increasing, and any strictly increasing ASF is also increasing. The following theorems define necessary and sufficient conditions for an optimal solution of (1) to be (weakly) Pareto optimal:

Theorem 1 Wierzbicki (1986a,b)

1. Let $s_{R}$ be strongly (strictly) increasing. If $x^{*} \in X$ is an optimal solution of problem (1), then $x^{*}$ is (weakly) Pareto optimal.

2. If $s_{R}$ is increasing and the solution of (1) $x^{*} \in X$ is unique, then $x^{*}$ is Pareto optimal.

Theorem 2 Miettinen (1999) If $s_{R}$ is strictly increasing and $x^{*} \in X$ is weakly Pareto optimal, then it is a solution of (1) with $f^{R}=f\left(x^{*}\right)$ and the optimal value of $s_{R}$ is zero.

The advantage of ASFs is that any (weakly) Pareto optimal solution can be obtained by moving the reference point only. It was shown in Wierzbicki (1986a) that the solution of an ASF depends Lipschitz continuously on the reference point. In general, ASFs are conceptually very appealing to generate Pareto optimal solutions, and they overcome most of the difficulties arising with other methods (Miettinen 1999) in the class of methods for generating Pareto optimal solutions.

The most well-known strictly increasing ASF is of Chebyshev type:

$$
s_{R}^{\infty}(f(x), \lambda)=\max _{i \in N_{m}} \lambda_{i}\left(f_{i}(x)-f_{i}^{R}\right),
$$

where $\lambda$ is $m$-vector of non-negative coefficients used for scaling purposes, that is, for normalizing objective functions of different magnitudes.

Note that here we do not use absolute value because we want to ensure that (weakly) Pareto optimal solutions are produced independently of the attainability or unattainability of the reference point. Indeed, we are in fact seeking for solutions which either minimize the distance from the reference point to the feasible region, if the reference point is unattainable, or maximize the distance otherwise.

The most well-known strongly increasing ASF is of augmented Chebyshev type:

$$
s_{R}^{\infty+1}(f(x), \lambda)=\rho \sum_{i \in N_{m}} \lambda_{i}\left(f_{i}(x)-f_{i}^{R}\right)+\max _{i \in N_{m}} \lambda_{i}\left(f_{i}(x)-f_{i}^{R}\right),
$$

where $\rho>0$ is a small parameter. 
Note that (3) can be viewed as a parameterized version of $s_{R}^{\infty}$ with a continuous parameter $\rho>0$. While the main term $\max _{i \in N_{m}} \lambda_{i}\left(f_{i}(x)-f_{i}^{R}\right)$ produces weakly Pareto optimal solutions, the augmented term $\rho \sum_{i \in N_{m}} \lambda_{i}\left(f_{i}(x)-f_{i}^{R}\right)$ is used at the same time to guarantee proper Pareto optimality of the obtained solutions (for the definition of proper Pareto optimality see, e.g. Miettinen 1999). In terms of Wierzbicki (1977), functions (2) and (3) are called order-representing and order-approximation functions, respectively. There are many variants and refinements of these ASFs which have been designed to guarantee Pareto optimality (Kaliszewski 1994; Miettinen and Mäkelä 2002).

Among others, some questions concerning ASFs based on the $L_{1}$ metric are discussed in Ruiz et al. (2008) and an additive ASF based on the $L_{1}$ metric is proposed as

$$
\hat{s}_{R}^{1}(f(x), \lambda)=\sum_{i \in N_{m}} \lambda_{i}\left|f_{i}(x)-f_{i}^{R}\right|
$$

It is evident that

$$
s_{R}^{\infty+1}(f(x), \lambda)=\rho \hat{s}_{R}^{1}(f(x), \lambda)+s_{R}^{\infty}(f(x), \lambda)
$$

in the case where the reference point dominates or is equal to the ideal solution.

Notice that (4) requires the reference point not to be strictly dominated by any feasible point in order to work properly. However, even in the case of non-dominated reference point, the solution produced may be not the best one due to excessive penalization of "good" (negative) deviations (for more details see Fig. 2 in Ruiz et al. (2008)). Penalizing "good" deviations makes no sense unless the reference point is attainable. To overcome the last-mentioned drawback, a modified additive ASF based on the $L_{1}$ metric is proposed in Ruiz et al. (2008) in the following form:

$$
s_{R}^{1}(f(x), \lambda)=\sum_{i \in N_{m}} \max \left[\lambda_{i}\left(f_{i}(x)-f_{i}^{R}\right), 0\right] .
$$

Note that $s_{R}^{1}(f(x), \lambda) \geq 0$. This function is still sensitive to the location of the reference point (i.e. being nondominated by any feasible point); however, it maintains a better penalization mechanism. Indeed, it allows penalizing "bad" (positive) deviations from the reference point but at the same time forbids penalizing "good" (negative) deviation.

The following properties of $s_{R}^{1}(f(x), \lambda)$ were proved in Ruiz et al. (2008).

Theorem 3 Ruiz et al. (2008) Given problem (1) with ASF defined by (5), let $f^{R}$ be a reference point such that $f^{R}$ is not dominated by an objective vector of any feasible solution of problem (1). Also assume $\lambda_{i}>0$ for all $i \in N_{m}$. Then any optimal solution of problem (1) is a weakly Pareto optimal solution.

It is easy to see by checking the proof in Ruiz et al. (2008) (see Theorem 2) that the result stated above is also valid in the case if the reference point is not strictly 
dominated by an objective vector of any feasible solution of problem (1), i.e., if there exist no feasible $x \in X$ with $f_{i}(x)<f_{i}^{R}$ for all $i \in N_{m}$.

Theorem 4 Ruiz et al. (2008) Given problem (1) with ASF defined by (5) and any reference point $f^{R}$, assume $\lambda_{i}>0$ for all $i \in N_{m}$. Then among the optimal solutions of problem (1) there exists at least one Pareto optimal solution. If the optimal solution of problem (1) is unique, then it is Pareto optimal.

Based on the above results, we may conclude that $s_{R}^{1}(f(x), \lambda)$ is sensitive to the correct choice of the reference point $f^{R}$, i.e., some sort of underestimation of aspiration levels is required from the DM to guarantee weak Pareto optimality. In case we want to guarantee Pareto optimality, an augmentation term can be used as in (3). Notice that earlier we mentioned ASFs that work for any kind of reference point, whereas $s_{R}^{1}(f(x), \lambda)$ is sensitive to the choice of a reference point. The assumption that the reference point should not be dominated by an objective vector of any feasible solution is the price the DM has to pay if (s)he wants to minimize the aggregated deviations from the reference point. Nevertheless, the good fact about $s_{R}^{1}(f(x), \lambda)$ is that, under the same set of weighting coefficients, it allows producing solutions which are significantly different from those produced by "classical" ASFs. These solutions represent a different preference structure and therefore could be a good alternative to those solutions obtained based on $s_{R}^{\infty}(f(x), \lambda)$.

In the following section we introduce a parameterized ASF, depending on a parameter $q \in N_{m}$, whose extreme cases coincide with $s_{R}^{1}(f(x), \lambda)$ for $q=1$, and $s_{R}^{\infty}(f(x), \lambda)$ for $q=m$, respectively.

\section{Parameterized achievement scalarizing functions}

In this section we extend ideas of Ruiz et al. (2008) by introducing a parameterization based on the notion of embedded subsets. We introduce an integer parameter $q \in N_{m}$ in order to control the degree of metric flexibility varying from $L_{1}$ to $L_{\infty}$.

Let $I^{q}$ be a subset of $N_{m}$ of cardinality $q$. A parameterized ASF is defined as follows:

$$
\tilde{s}_{R}^{q}(f(x), \lambda)=\max _{I^{q} \subseteq N_{m}:\left|I^{q}\right|=q}\left\{\sum_{i \in I^{q}} \max \left[\lambda_{i}\left(f_{i}(x)-f_{i}^{R}\right), 0\right]\right\},
$$

where $q \in N_{m}$ and $\lambda=\left(\lambda_{1}, \ldots, \lambda_{m}\right), \lambda_{i}>0, i \in N_{m}$. Notice that

- for $q \in N_{m}: s_{R}^{q}(f(x), \lambda) \geq 0$;

- for $q=1: \tilde{s}_{R}^{1}(f(x), \lambda)=\max _{i \in N_{m}} \max \left[\lambda_{i}\left(f_{i}(x)-f_{i}^{R}\right), 0\right] \cong s_{R}^{\infty}(f(x), \lambda)$;

- for $q=m: \tilde{s}_{R}^{m}(f(x), \lambda)=\sum_{i \in N_{m}} \max \left[\lambda_{i}\left(f_{i}(x)-f_{i}^{R}\right), 0\right]=s_{R}^{1}(f(x), \lambda)$.

Here, "" means equality in the case where there exist no feasible solutions $x \in X$ which strictly dominate the reference point, i.e., such that $f_{i}(x)<f_{i}^{R}$ for all $i \in N_{m}$. 
The problem to be solved is

$$
\min _{x \in X} \tilde{s}_{R}^{q}(f(x), \lambda)
$$

It is obvious that using problem (7), every feasible solution of the multiobjective problem (including Pareto optimal) is supported. Indeed, given any $x \in X$, the reference point $f^{R}=f(x)$ and a vector of weighting coefficients $\lambda>0$, the optimal solution to problem (7) is $x$ with the optimal value of $\tilde{s}_{R}^{q}(f(x), \lambda)$ equals zero. Thus, the first of the two requirements, mentioned in Sect. 2, holds.

For any $x \in X$, denote $I_{x}=\left\{i \in N_{m}: f_{i}^{R} \leq f_{i}(x)\right\}$. The following result is analogous to Theorem 3:

Theorem 5 Given problem (7), let $f^{R}$ be a reference point such that there exists no feasible solution whose image strictly dominates $f^{R}$. Also assume $\lambda_{i}>0$ for all $i \in N_{m}$. Then, any optimal solution of problem (7) is a weakly Pareto optimal solution.

Proof The proof will be given by contradiction. Let $x^{*}$ be an optimal solution of problem (7). We assume that $x^{*}$ is not weakly Pareto optimal. Then, there exists a feasible solution $x^{\prime} \in X$ such that $f_{i}\left(x^{\prime}\right)<f_{i}\left(x^{*}\right)$ for all $i \in N_{m}$.

Notice that $I_{x^{\prime}} \subseteq I_{x^{*}}$, and $I_{x^{\prime}} \neq \emptyset$ under the assumption that there exists no feasible solution whose image strictly dominates the reference point. Then,

$$
\begin{aligned}
\tilde{s}_{R}^{q}\left(f\left(x^{\prime}\right), \lambda\right) & =\max _{I^{q} \subseteq N_{m}:\left|I^{q}\right|=q}\left\{\sum_{i \in I^{q}} \max \left[\lambda_{i}\left(f_{i}\left(x^{\prime}\right)-f_{i}^{R}\right), 0\right]\right\} \\
& =\max _{I^{q} \subseteq N_{m}:\left|I^{q}\right|=q}\left\{\sum_{i \in I^{q} \cap I_{x^{\prime}}} \lambda_{i}\left(f_{i}\left(x^{\prime}\right)-f_{i}^{R}\right)\right\} \\
& <\max _{I^{q} \subseteq N_{m}:\left|I^{q}\right|=q}\left\{\sum_{i \in I^{q} \cap I_{x^{\prime}}} \lambda_{i}\left(f_{i}\left(x^{*}\right)-f_{i}^{R}\right)\right\} \\
& \leq \max _{I^{q} \subseteq N_{m}:\left|I^{q}\right|=q}\left\{\sum_{i \in I^{q} \cap I_{x^{*}}} \lambda_{i}\left(f_{i}\left(x^{*}\right)-f_{i}^{R}\right)\right\} \\
& =\max _{I^{q} \subseteq N_{m}:\left|I^{q}\right|=q}\left\{\sum_{i \in I^{q}} \max \left[\lambda_{i}\left(f_{i}\left(x^{*}\right)-f_{i}^{R}\right), 0\right]\right\}=\tilde{s}_{R}^{q}\left(f\left(x^{*}\right), \lambda\right) .
\end{aligned}
$$

The obtained inequality $\tilde{s}_{R}^{q}\left(f\left(x^{\prime}\right), \lambda\right)<\tilde{s}_{R}^{q}\left(f\left(x^{*}\right), \lambda\right)$ contradicts the assumption of $x^{*}$ being an optimal solution of problem (7). This completes the proof.

In the way similar to the proof of Theorem 4 (Theorem 1 in Ruiz et al. 2008) the following fact can be proven:

Theorem 6 Given problem (7), let $f^{R}$ be any reference point. Also assume $\lambda_{i}>0$ for all $i \in N_{m}$. Then, among the optimal solutions of problem (7) there exists at least one Pareto optimal solution. 
Theorem 6 implies that the uniqueness of the optimal solution guarantees its Pareto optimality. Notice that the facts stated above about solutions of parameterized ASFs also implicitly follow from the results of Theorem 1 . To show this, it is sufficient to prove that $\tilde{s}_{R}^{q}(f(x), \lambda)$ is increasing. Moreover, it is strictly increasing if there are no feasible solutions strictly dominating $f^{R}$.

Indeed, take $x_{1} \in X$ and $x_{2} \in X$ with $f_{i}\left(x_{1}\right) \leq f_{i}\left(x_{2}\right)$ for all $i \in N_{m}$. Since $\lambda_{i}>0$ for all $i \in N_{m}$, then

$$
\begin{aligned}
\tilde{s}_{R}^{q}\left(f\left(x_{1}\right), \lambda\right) & =\max _{I^{q} \subseteq N_{m}:\left|I^{q}\right|=q}\left\{\sum_{i \in I^{q}} \max \left[\lambda_{i}\left(f_{i}\left(x_{1}\right)-f_{i}^{R}\right), 0\right]\right\} \\
& \leq \max _{I^{q} \subseteq N_{m}:\left|I^{q}\right|=q}\left\{\sum_{i \in I^{q}} \max \left[\lambda_{i}\left(f_{i}\left(x_{2}\right)-f_{i}^{R}\right), 0\right]\right\}=\tilde{s}_{R}^{q}\left(f\left(x_{2}\right), \lambda\right),
\end{aligned}
$$

i.e., $\tilde{s}_{R}^{q}(f(x), \lambda)$ is increasing.

Moreover, if we take $x_{1} \in X$ and $x_{2} \in X$ with $f_{i}\left(x_{1}\right)<f_{i}\left(x_{2}\right)$ for all $i \in N_{m}$, then, provided that there exists no solution $x \in X$ with $f_{i}(x)<f_{i}^{R}$ for all $i \in N_{m}$, we get $I_{x_{1}} \subseteq I_{x_{2}}$ and $I_{x_{1}} \neq \emptyset$, and thus, recalling that $\lambda_{i}>0$ for all $i \in N_{m}$, we deduce the following:

$$
\begin{aligned}
\tilde{s}_{R}^{q}\left(f\left(x_{1}\right), \lambda\right) & =\max _{I^{q} \subseteq N_{m}:\left|I^{q}\right|=q}\left\{\sum_{i \in I^{q}} \max \left[\lambda_{i}\left(f_{i}\left(x_{1}\right)-f_{i}^{R}\right), 0\right]\right\} \\
& =\max _{I^{q} \subseteq N_{m}:\left|I^{q}\right|=q}\left\{\sum_{i \in I^{q} \cap I_{x_{1}}} \lambda_{i}\left(f_{i}\left(x_{1}\right)-f_{i}^{R}\right)\right\} \\
& <\max _{I^{q} \subseteq N_{m}:\left|I^{q}\right|=q}\left\{\sum_{i \in I^{q} \cap I_{x_{2}}} \lambda_{i}\left(f_{i}\left(x_{2}\right)-f_{i}^{R}\right)\right\} \\
& =\max _{I^{q} \subseteq N_{m}:\left|I^{q}\right|=q}\left\{\sum_{i \in I^{q}} \max \left[\lambda_{i}\left(f_{i}\left(x_{2}\right)-f_{i}^{R}\right), 0\right]\right\}=\tilde{s}_{R}^{q}\left(f\left(x_{2}\right), \lambda\right),
\end{aligned}
$$

i.e., $\tilde{s}_{R}^{q}(f(x), \lambda)$ is strictly increasing.

Thus, Theorems 5 and 6 , describe the conditions under which the second of the two requirements, mentioned in Sect. 2, holds. So, we have now considered both major requirements.

Various Pareto optimal solutions can be detected not only by moving the reference point itself, but also by manipulating the weighting coefficients while a reference point is remaining fixed. In this paper, we consider the simplest case where weighting coefficients are fixed for scaling (i.e. normalizing). Alternatively, the weighting coefficients may, e.g., reflect the level of penalization for "bad" deviations which the DM wants to introduce into the problem. Different ways of reflecting preference information and manipulating weighting coefficients of parameterized ASFs may be a subject for future research similar to what has been done in Luque et al. (2009). 
Parameterized ASFs can be potentially used either for some particular values of $q$ or for all $q \in N_{m}$ simultaneously. The simplest but the most computationally demanding way is to calculate ASFs for all $q \in N_{m}$. Since the solution of a problem with a smaller $q$-value unlikely provides any help for the solution of the problem with a larger $q$-value and vice versa, it seems realistic to use parallel computation for these purposes. The choice of a particular $q$-value can be done if some extra information is available (at least locally) about the shape of the Pareto frontier as well as the shape of the $R$-level set (i.e. a set of points for which the distance from the reference point is equal to $R$ in terms of the corresponding ASF) of the parameterized ASF for the given $q$-value. In general, getting such information may be a very complicated task and practically can be potentially fulfilled under strong assumptions like, e.g., objective functions convexity etc.

Notice also that the choice of parameter $q$ affects directly the shape of $R$-level that may vary from being sharp in the case of $\tilde{s}_{R}^{1}(f(x), \lambda)$ to linearly flat as in the case of $\tilde{s}_{R}^{m}(f(x), \lambda)$. This helps the ASF to penetrate into the areas where lots of non-supported solutions are accumulated.

As in the case with the additive $\operatorname{ASF}_{R}^{1}(f(x), \lambda)$ developed in Ruiz et al. (2008), the parameterized $\operatorname{ASF} \tilde{s}_{R}^{q}(f(x), \lambda)$ inherits the similar limitation: one has to keep always in mind that the reference point should not be strictly dominated by some feasible point. However, if this is the case, the point which strictly dominates the reference point could be easily detected, and the problem can be overcame as pointed out in Ruiz et al. (2008).

Problem (7) is non-differentiable due to the presence of the min-max term. It can be solved, for example, with efficient bundle methods (see, e.g. Mäkelä 2002). However, if (7) is nonconvex, only local optima (with no guarantee of being global optima in general) can be produced with these methods. Instead, (7) can be turned into an integer differentiable form, which is suitable for any mixed-integer programming (MIP) solver (Westerlund and Pörn 2002), as follows:

$\min \alpha$

subject to

$$
\begin{aligned}
& \alpha \geq \sum_{i \in I_{s}^{q}} \lambda_{i}\left(1-z_{i}^{s}\right)\left(f_{i}(x)-f_{i}^{R}\right) \quad s=1, \ldots,\left(\begin{array}{c}
m \\
q
\end{array}\right) \\
& f_{i}^{R}-f_{i}(x) \leq z_{i}^{s} M \quad i \in I^{q}, s=1, \ldots,\left(\begin{array}{c}
m \\
q
\end{array}\right) \\
& f_{i}^{R}-f_{i}(x) \geq\left(z_{i}^{s}-1\right) M \quad i \in I^{q}, s=1, \ldots,\left(\begin{array}{c}
m \\
q
\end{array}\right) \\
& x \in X, \quad z_{i}^{s} \in\{0,1\}, \quad i \in N_{m}, s=1, \ldots,\left(\begin{array}{c}
m \\
q
\end{array}\right) .
\end{aligned}
$$

Here, $s$ is used to enumerate all $q$-element subsets $I_{s}^{q}$ of an $m$-element set $N_{m}$; for any $i \in I_{s}^{q}, z_{i}^{s}$ is a binary variable; $M$ is a sufficiently large number to ensure that $z_{i}^{s}=1$ iff $f_{i}^{R}-f_{i}(x)>0$, and $z_{i}^{s}=0$ iff $f_{i}^{R}-f_{i}(x) \leq 0$. It is easy to see that the optimal solution of problem (8) is the feasible solution that optimizes the parameterized ASF (6). However, the problem of finding a global optimum cannot be resolved easily in a general case. 
This mixed-integer programming model contains $4 \cdot\left(\begin{array}{c}m \\ q\end{array}\right)$ constraints, so one may expect the increase of computational time while $\left(\begin{array}{c}m \\ q\end{array}\right)$ grows up. A large number of constraints can be efficiently treated by a MIP solver if the constraint propagation mechanisms (see, e.g. Rossi et al. 2006) are incorporated. Instead, solvers not assuming differentiability can be used.

Despite increasing computational efforts, the parameterized ASFs present a new approach (based on parameterization) how to generate systematically different ASFs which may potentially produce different solutions with different $q$-values. Further understanding of how different $q$-values produce different shapes of $R$-level sets may shed extra light on the practical application of the parameterized ASFs. This could be a challenging and promising topic for further research. In the next section, we lightly treat this and other questions for the simplest case with three objective functions.

\section{Case of three objectives}

Because the formulation of the proposed ASF may seem rather complicated and to illustrate some ideas mentioned earlier, in this section we consider a special case of three objective functions, that is, $m=3$ and write explicitly what the ASFs look like in this situation. Then, (6) has the following form:

$$
\tilde{s}_{R}^{q}(f(x), \lambda)=\max _{I^{q} \subseteq\{1,2,3\}:\left|I^{q}\right|=q}\left\{\sum_{i \in I^{q}} \max \left[\lambda_{i}\left(f_{i}(x)-f_{i}^{R}\right), 0\right]\right\},
$$

where $q=1,2,3$ and $\lambda=\left(\lambda_{1}, \lambda_{2}, \lambda_{3}\right), \lambda_{i}>0, i \in N_{3}$.

In other words, we have

for $q=1$

$$
\begin{aligned}
\tilde{s}_{R}^{1}(f(x), \lambda)=\max \{ & \max \left[\lambda_{1}\left(f_{1}(x)-f_{1}^{R}\right), 0\right], \max \left[\lambda_{2}\left(f_{2}(x)-f_{2}^{R}\right), 0\right], \\
\left.\max \left[\lambda_{3}\left(f_{3}(x)-f_{3}^{R}\right), 0\right]\right\} & \\
=\max \{ & \lambda_{1} \max \left[f_{1}(x)-f_{1}^{R}, 0\right], \lambda_{2} \max \left[f_{2}(x)-f_{2}^{R}, 0\right], \\
& \left.\lambda_{3} \max \left[f_{3}(x)-f_{3}^{R}, 0\right]\right\} ;
\end{aligned}
$$

for $q=2$

$$
\begin{aligned}
\tilde{s}_{R}^{2}(f(x), \lambda)=\max \quad\{ & \max \left[\lambda_{1}\left(f_{1}(x)-f_{1}^{R}\right), 0\right]+\max \left[\lambda_{2}\left(f_{2}(x)-f_{2}^{R}\right), 0\right], \\
& \max \left[\lambda_{1}\left(f_{1}(x)-f_{1}^{R}\right), 0\right]+\max \left[\lambda_{3}\left(f_{3}(x)-f_{3}^{R}\right), 0\right], \\
& \left.\max \left[\lambda_{2}\left(f_{2}(x)-f_{2}^{R}\right), 0\right]+\max \left[\lambda_{3}\left(f_{3}(x)-f_{3}^{R}\right), 0\right]\right\} \\
=\max \quad\left\{\lambda_{1} \max \left[f_{1}(x)-f_{1}^{R}, 0\right]+\lambda_{2} \max \left[f_{2}(x)-f_{2}^{R}, 0\right],\right. & \lambda_{1} \max \left[f_{1}(x)-f_{1}^{R}, 0\right]+\lambda_{3} \max \left[f_{3}(x)-f_{3}^{R}, 0\right], \\
& \left.\lambda_{2} \max \left[f_{2}(x)-f_{2}^{R}, 0\right]+\lambda_{3} \max \left[f_{3}(x)-f_{3}^{R}, 0\right]\right\} ;
\end{aligned}
$$


Fig. 1 1-level set for $\tilde{s}_{R}^{1}(f(x), \lambda)$

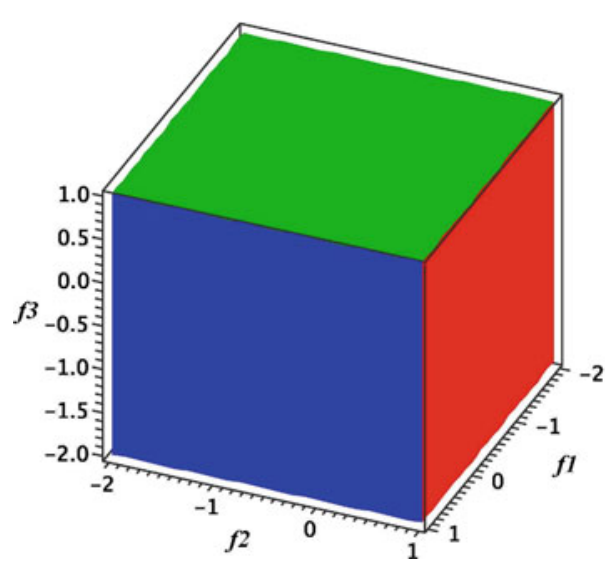

for $q=3$

$$
\begin{aligned}
\tilde{s}_{R}^{3}(f(x), \lambda)= & \max \left[\lambda_{1}\left(f_{1}(x)-f_{1}^{R}\right), 0\right] \\
& +\max \left[\lambda_{2}\left(f_{2}(x)-f_{2}^{R}\right), 0\right]+\max \left[\lambda_{3}\left(f_{3}(x)-f_{3}^{R}\right), 0\right] \\
= & \lambda_{1} \max \left[f_{1}(x)-f_{1}^{R}, 0\right]+\lambda_{2} \max \left[f_{2}(x)-f_{2}^{R}, 0\right] \\
& +\lambda_{3} \max \left[f_{3}(x)-f_{3}^{R}, 0\right] .
\end{aligned}
$$

For calculating examples which appear later in this section we used MAPLE 11 software with the built-in solver which uses sequential quadratic programming methods (see, e.g. Fletcher 1980).

Now we give a graphical interpretation of level sets in our 3-dimensional space. To simplify illustration, we restrict the view inside the image space within a rectangular $\left\{f=\left(f_{1}, f_{2}, f_{3}\right)^{T}:-2 \leq f_{i} \leq 1, i \in N_{3}\right\}$. Let us assume that the reference point is the origin, i.e., $f^{R}=(0,0,0)^{T}$. Assume also that all objective functions are identity mappings, i.e., we can operate in objective space only. We are interested in depicting a 1-level set, i.e., a set of points $\left(f_{1}, f_{2}, f_{3}\right)^{T}$ for which the distance from the reference point (coordinate origin) is equal to 1 with respect to the corresponding ASF. The case when all weighting coefficients are equal to one, i.e., $\lambda_{1}=\lambda_{2}=\lambda_{3}=1$, is considered. Figures 1,2 and 3 shows the 1 -sets for $\tilde{s}_{R}^{1}(f, \lambda), \tilde{s}_{R}^{2}(f, \lambda)$ and $\tilde{s}_{R}^{3}(f, \lambda)$, respectively. While the 1 -level set for $\tilde{s}_{R}^{1}(f, \lambda)$ looks very simple and similar to what we always have in the case of the Chebyshev type ASF, the constructions of 1-level sets for $\tilde{s}_{R}^{2}(f, \lambda)$ and $\tilde{s}_{R}^{3}(f, \lambda)$ are more sophisticated.

Let us look at the constructions in more detail. Those faces which are parallel to the faces $f_{1} f_{2}, f_{1} f_{3}$, or $f_{2} f_{3}$ are formed if one of the three maxima equals to one, while the other two are less than one or equal to zero. Those faces which are sloped and parallel to the coordinate rays are formed if a sum of two of the three maxima equals to one, while the third one is less than one or equal to zero. The difference between 1-level sets for $\tilde{s}_{R}^{2}(f, \lambda)$ and $\tilde{s}_{R}^{3}(f, \lambda)$ lies in constructing of faces that correspond to the case where all three maxima are positive and their sum has to be equal to one. For $\tilde{s}_{R}^{3}(f, \lambda)$, this forms a flat triangle face (see Fig. 4 ), whereas for $\tilde{s}_{R}^{2}(f, \lambda)$ a flat triangle 
Fig. 2 1-level set for $\tilde{s}_{R}^{2}(f(x), \lambda)$

Fig. 3 1-level set for $\tilde{s}_{R}^{3}(f(x), \lambda)$

Fig. 4 Zooming 1-level set for $\tilde{s}_{R}^{3}(f(x), \lambda)$
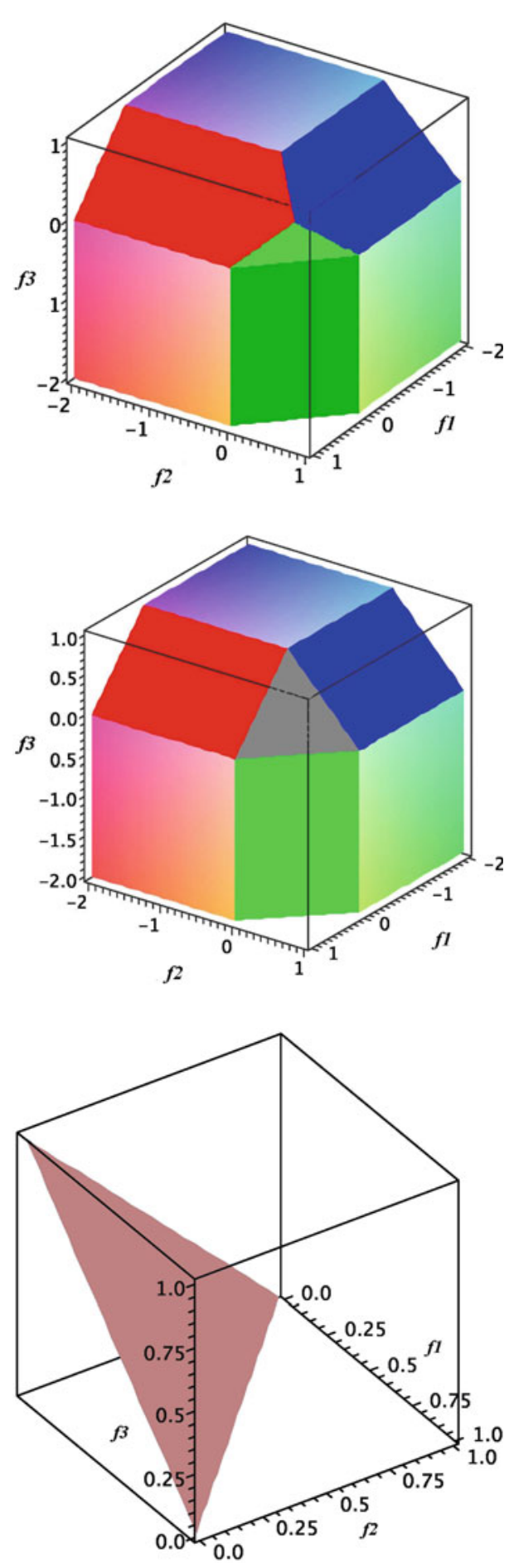
Fig. 5 Zooming 1-level set for $\tilde{s}_{R}^{2}(f(x), \lambda)$

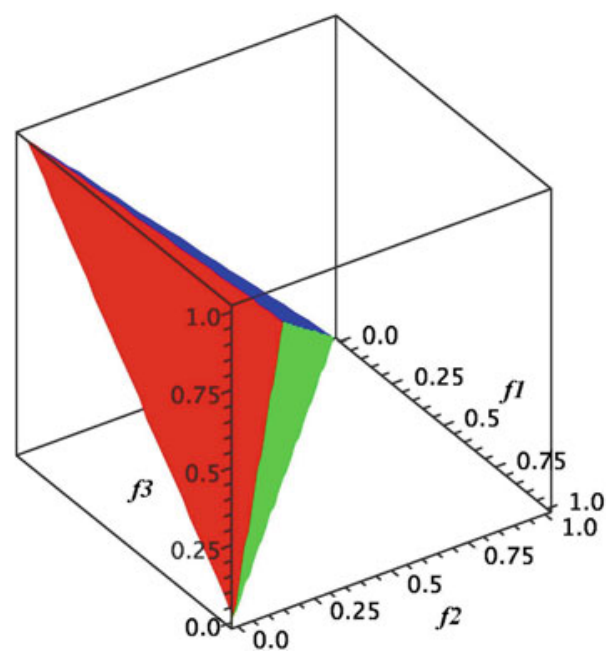

transforms into a triangle pyramid with a top vertex $\left(\frac{1}{2}, \frac{1}{2}, \frac{1}{2}\right)$ (see, Fig. 5). This top vertex corresponds to the case when all three maxima participating in $\tilde{s}_{R}^{2}(f, \lambda)$ are equal to $\frac{1}{2}$, and hence adding any two of them will give us distance one.

The natural question which may arise is the following: why do we need different ASFs? Is it not enough to have one ASF only? We can answer this question by establishing some parallels between multiobjective optimization and art. For example, in art drawing, a painter may need a large variety of brushes (different in size, form etc) to deal with the most delicate details of her/his painting, e.g., to draw one small point without touching and disturbing neighboring points. The situation looks similar to multiobjective optimization where, if a DM wants to detect one particular point (maybe with some pre-specified properties) on the Pareto front, she/he has to vary the reference point or, alternatively, may need a different shape of $R$-level sets of the ASF to perform this task correctly. Certainly, the question which shape of $R$-level sets of the ASF could be the best fit is generally quite complicated, so the answer may require additional computational efforts or extra knowledge about the structure of the Pareto frontier.

One more issue which has to be emphasized is that (as it can be seen from Fig. 4), the linear (flat triangle) part of the $R$-level becomes larger when $R$ is increasing, i.e., when the reference point is moving far from the place of potential contact of $R$-level with the image of the feasible set. Accordingly, the penetrating of the additive ASF towards the location of non-supported solutions may be complicated, while the parameterized ASF with $q \neq m$ may show better performance in this case.

One more reason why using various ASFs may be potentially advantageous is the following. The efficiency of some interactive methods (see, e.g. Miettinen et al. 2008; Miettinen 1999 for more details about interactive methods in multiobjective optimization) can be increased while different variants of ASFs are used producing different but still 'good' solutions. For example, the idea of formulating several ASFs, all using the same preference information from the DM, is efficiently used in the synchronous NIMBUS approach presented in Miettinen and Mäkelä (2006). Under this 
approach, the method developers do not make the choice between different ASFs but calculate the results of different ASFs and leave the final decision to the DM. The idea of using the parameterized ASF could be implemented as follows: once $\tilde{s}_{R}^{1}(f(x), \lambda)$ and $\tilde{s}_{R}^{m}(f(x), \lambda)$ produced solutions, which are significantly different, the middle point with respect to $q$ parameterized $\operatorname{ASF} \tilde{s}_{R}^{\left\lfloor\frac{m+1}{2}\right\rfloor}(f(x), \lambda)$ is used to calculate one more solution to be compared with the two already produced. Such dichotomy is continued until the solutions produced become insignificantly different or just the same. Thus, a larger variety of significantly different solutions can be produced under the same preference information.

The following example shows both graphically and numerically that the solutions obtained by means of $\tilde{s}_{R}^{q}(f(x), \lambda)$ are generally different for all $q \in N_{m}$ : Let us minimize

$$
\begin{aligned}
f(x) & =\left(f_{1}(x), f_{2}(x), f_{3}(x)\right) \\
& =\left(\left(x_{1}+1\right)^{2}+\left(x_{2}-5\right)^{2},\left(x_{1}-3\right)^{2}+\left(x_{2}+3\right)^{2},\left(x_{1}-4\right)^{2}+\left(x_{2}+2\right)^{2}\right)
\end{aligned}
$$

subject to

$$
\begin{aligned}
& x_{1}+2 x_{2} \leq 10 \\
& 1 \leq x_{1} \leq 10,1 \leq x_{2} \leq 4
\end{aligned}
$$

The ideal objective vector is $f^{I}=(5,16,9)$, which is also assumed to be selected as a reference point $f^{R}$. We define weighting coefficients in a standard way to provide objective normalization: $\lambda_{1}=\frac{1}{f_{1}^{I}}, \lambda_{2}=\frac{1}{f_{2}^{I}}, \lambda_{3}=\frac{1}{f_{3}^{I}}$, i.e., $\lambda=(0.20000,0.06250,0.11111)$.

Then the optimal solutions of (7) for different values of $q \in N_{3}$ are the following:

$$
\begin{aligned}
x^{\prime} & =\arg \min _{x \in X} \tilde{s}_{R}^{1}(f(x), \lambda)=(1.13525,2.01064), \tilde{s}_{R}^{1}\left(f\left(x^{\prime}\right), \lambda\right)=1.69911, \\
f\left(x^{\prime}\right) & =(13.49556,28.58381,24.29202) ; \\
x^{\prime \prime} & =\arg \min _{x \in X} \tilde{s}_{R}^{2}(f(x), \lambda)=(1.00000,2.44653), \tilde{s}_{R}^{2}\left(f\left(x^{\prime \prime}\right), \lambda\right)=3.30089, \\
f\left(x^{\prime \prime}\right) & =(10.52021,33.66468,28.77162) ; \\
x^{\prime \prime \prime} & =\arg \min _{x \in X} \tilde{s}_{R}^{1}(f(x), \lambda)=(1.57993,1.15613), \tilde{s}_{R}^{3}\left(f\left(x^{\prime \prime \prime}\right), \lambda\right)=4.11524, \\
f\left(x^{\prime \prime \prime}\right) & =(16.34582,24.37556,20.90344) .
\end{aligned}
$$

These three optimal solutions together with the ideal objective vector (the black point outside the feasible region) are depicted in Fig. 6. Note that the solution $f\left(x^{\prime \prime}\right)$ is significantly different from $f\left(x^{\prime \prime \prime}\right)$ and $f\left(x^{\prime}\right)$, and it cannot be obtained easily from the other two, e.g., by a linear combination.

It is clear that different ASFs may have different computational costs (see e.g. Miettinen et al. 2006). It is also obvious that using extra ASFs within interactive methods may lead to the increase of computational efforts at each iteration of the interactive process. However, if available, parallel computing can be exploited, since 
Fig. 6 Ideal vector and three different solutions produced by $\tilde{s}_{R}^{1}(f(x), \lambda), \tilde{s}_{R}^{2}(f(x), \lambda)$, $\tilde{s}_{R}^{3}(f(x), \lambda)$

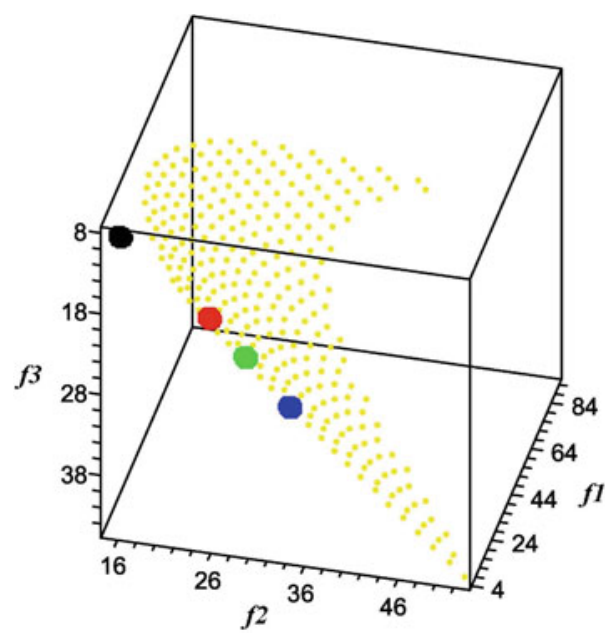

different ASFs are independent of each others. At the same time, using ASFs simultaneously may reduce the number of iterations needed for the iterative solution process to converge, i.e., to find a solution which is most preferred by the DM. Reducing the number of iterations may decrease the total time of the interactive process, since exchanging information with the DM is sometimes much more time consuming than the optimization itself.

\section{Conclusion}

In this paper we have proposed an approach how to parameterize achievement scalarizing functions based on the introduction of a discrete integer parameter which varies from 1 to $m$, where $m$ represents the number of objectives. We have proven the ability of the parameterized ASF to produce weakly Pareto optimal solutions assuming the reference point is selected to be non-dominated by any feasible solution.

We have illustrated that the solutions obtained by parameterized ASFs may be significantly different. This fact could be potentially exploited in interactive processes to speed up their convergence in terms of the number of iterations, and provide the DM with more flexible tools to detect desirable Pareto optimal points. This gives us some confidence that the parameterized ASFs may find their applications in synchronous approaches, i.e., those using several different ASFs at once in a systematic way. As prospective research, we would like to consider applicability of parameterized ASFs to the well-known interactive methods, e.g. the NIMBUS method (Miettinen and Mäkelä 2006).

\section{References}

Deb K, Miettinen K (2010) Nadir point estimation using evolutionary approaches: better accuracy and computational speed through focused search. In: Ehrgott M et al (eds) Multiple criteria decision making for sustainable energy and transportation system. Springer, Berlin, pp 339-354 
Ehrgott M (2000) Multicriteria optimization. Springer, Berlin

Fletcher R (1980) Practical methods of optimization. Wiley, New York

Kaliszewski I (1994) Quantitative Pareto analysis by cone separation technique. Kluwer Academic Publishers, Dordrecht

Luque M, Miettinen K, Eskelinen P, Ruiz F (2009) Incorporating preference information in interactive reference point methods for multiobjective optimization. Omega 37:450-462

Luque M, Ruiz F, Miettinen K (2009) Global formulation for interactive multiobjective optimization, OR Spectrum. doi:10.1007/s00291-008-0154-3

Miettinen K (1999) Nonlinear multiobjective optimization. Kluwer Academic Publishers, Boston

Miettinen K, Ruiz F, Wierzbicki AP (2008) Introduction to multiobjective optimization: interactive approaches. In: Branke $\mathrm{J}$ et al (eds) Multiobjective optimization interactive and evolutionary approaches. Lecture Notes in computer science, vol 5252. Springer, Berlin, pp 27-58

Miettinen K, Mäkelä MM (2006) Synchronous approach in interactive multiobjective optimization. Eur J Oper Res 170(3):909-922

Miettinen K, Mäkelä MM (2002) On scalarizing functions in multiobjective optimization. OR Spectr 24:193-213

Miettinen K, Mäkelä MM, Kaario K (2006) Experiments with classification-based scalarizing functions in interactive multiobjective optimization. Eur J Oper Res 175(2):931-947

Mäkelä MM (2002) Survey of bundle methods for nonsmooth optimization. Optim Methods Softw 17(1): $1-20$

Pareto V (1909) Manuel d'ecoonomie politique. Qiard, Paris

Rossi F, van Beek P, Walsh T (eds) (2006) Handbook of constraint programming. Elsevier

Ruiz F, Luque M, Miguel F, del Mar Muñoz M (2008) An additive achievement scalarizing function for multiobjective programming problems. Eur J Oper Res 188(3):683-694

Sawaragi Y, Nakayama H, Tanino T (1985) Theory of multiobjective optimization. Academic Press, Orlando

Slater M (1950) Lagrange multipliers revisited. Cowles Commission Discussion Paper: Mathematics 403

Steuer R (1986) Multiple criteria optimization: theory, computation and application. Wiley, New York

Westerlund T, Pörn R (2002) Solving pseudo-convex mixed-integer optimization problems by cutting plane techniques. Optim Eng 3:253-280

Wierzbicki AP (1977) Basic properties of scalarizing functionals for multiobjective optimization. Optimization 8:55-60

Wierzbicki AP (1980) The use of reference objectives in multiobjective optimization. In: Fandel G, Gal T (eds) Multiple criteria decision making theory and applications. MCDM theory and applications proceedings. Lecture notes in economics and mathematical systems, vol 177. Springer, Berlin, pp 468-486

Wierzbicki AP (1986a) A methodological approach to comparing parametric characterizations of efficient solutions. In: Fandel G et al (eds) Large-scale modelling and interactive decision analysis. Lecture notes in economics and mathematical systems, vol 273. Springer, Berlin, pp 27-45

Wierzbicki AP (1986b) On the completeness and constructiveness of parametric characterizations to vector optimization problems. OR Spectr 8:73-87

Wierzbicki AP (1999) Reference point approaches. In: Gal T et al (eds) Multicriteria decision making: advances in MCDM models, algorithms, theory, and applications. Kluwer, Boston, pp 1-39 